\title{
Optical properties of metal nanoparticles with arbitrary shapes
}

\author{
Iván O. Sosa, Cecilia Noguez ${ }^{\dagger}$ and Rubén G. Barrera ${ }^{\ddagger}$ \\ Instituto de Física, Universidad Nacional Autónoma de México, \\ Apartado Postal 20-364, Distrito Federal 01000, México
}

April 5, 2019

\begin{abstract}
We have studied the optical properties of metallic nanoparticles with arbitrary shape. We performed theoretical calculations of the absorption, extinction and scattering efficiencies, which can be directly compared with experiments, using the Discrete Dipole Approximation (DDA). In this work, the main features in the optical spectra have been investigated depending of the geometry and size of the nanoparticles. The origin of the optical spectra are discussed in terms of the size, shape and material properties of each nanoparticle, showing that a nanoparticle can be distinguish by its optical signature.
\end{abstract}

\footnotetext{
${ }^{*}$ E-mail: ivan@fisica.unam.mx

${ }^{\dagger}$ Author to whom all correspondence should be addressed. E-mail: cecilia@fisica.unam.mx

${ }^{\ddagger}$ E-mail: rbarrera@fisica.unam.mx
} 


\section{Introduction}

The fabrication of nanostructures requires a deep understanding of the physical phenomena involved at this length-scale. Low-dimensional quantum structures have shown to have unique optical and electronic properties. In particular, the shape and size of low-dimensional structures are crucial parameters that determine those physical properties. The characterization of these parameters are important issues either in fundamental research or in technological applications, covering from growth and characterization to device processing. Among nanostructures, metallic nanoparticles are also important because some of their main physical properties might be completely different from the corresponding ones in either molecules or bulk solids. For example, they might assume crystal structures that do not correspond to those of the bulk solid. $[1,2]$. Also, the catalytic activity of some of them depends strongly on their size and shape.

Typically, the estimation of the shape and size of nanoparticles has been done using techniques like Atomic Force Microscopy (AFM), Scanning Tunneling Microscopy (STM), Transmission Electron Microscopy (TEM) and Reflection High-Energy Electron Diffraction (RHEED) $[2,5,6,7,8]$. These techniques provide the image of a small piece of the sample, this means that they give information about local properties by characterizing a few nanoparticles at a time. Different shapes of nanoparticles have been reported with the use of these techniques, such as spheres, spheroids, lens-shaped, cone-shaped, pyramids with different facets, truncated pyramids, and different types of polyhedra $[9,10,11,2]$. A vast amount of information has been obtained through these "structural-characterization techniques", however, they still have some limitations. One limitation is that in most cases the growth and characterization are made in different ambients, which is a serious problem because the properties of nanoparticles are ambient dependent. In some of these techniques the sample is literally touched during characterization and sometimes this might substantially modify the properties of a nanoparticle. Furthermore, the growth and characterization of nanoparticles are usually made at different times, and this might become an additional uncontrollable variable.

The limitations of these "structural-characterization techniques" make desirable the use 
of complementary tools that could accomplish the same objectives but in the same ambient, in real time, in a non-destructive way, and providing statistical properties of the whole sample. Among these complementary tools one finds that within this context optical spectroscopies have been extremely useful due to their non-destructive and real-time character together with their in situ potentiality $[12,13,14]$. The above attributes can be found, for example, in Light Absorption Spectroscopy, Surface Enhanced Raman Scattering (SERS), Differential Reflectance (DR), etc. $[12,13,14]$. These attributes have allowed to control the growth of superlattices [15], and with a proper implementation of them, it might be possible to control also the growth of nanoparticles, correcting their shape and size during the growth process. In the future, this fact will be of crucial importance for the development of nanosciences and their technological applications.

A variety of results using optical techniques have been able to relate the spectroscopic features of the nanoparticles spectra with the excitation of surface plasmons and excitons, as well as the significant enhancement in Raman-absorption -excitation peak intensities, with the size and shape of a given system $[12,16,17,20]$. These features are manifested in optical spectra such as in light absorption, reflection and transmission or in light-extinction and scattering spectra, however, a clear physical interpretation of this spectroscopic information is still waiting. In conclusion, the actual correct determination of the size and shape parameters of a given nanoparticle is still controversial because a more complete experimental determination is needed, together with a corresponding clear physical interpretation.

In the present work, we study the optical properties of metallic nanometer-sized particles with different shapes and sizes. We calculate and discuss spectra for the extinction, absorption and scattering efficiencies. In particular, we show results for gold and silver nanoparticles. Our main goal is twofold, first we show how the main peaks of the optical spectra can be associated to the shape and size of the nanoparticle, as well as its material properties, and second we show the relative importance of absorption and scattering processes as a function of the same geometrical and material parameters. Although our calculation procedure is, at the present time, unable to display the actual fields induced in the particle, we can attach a multipolar character to the different excitations responsible for the charateristic features of the spectra by comparing results for particles with the same 
shape but with different size. We have also paid a particular attention to the accuracy of our calculations showing explicitly the conditions under which they might become numerically unstable, and comparing them with similar calculations performed by other authors. We believe that this study can be very useful to determine and optimize some of the physical properties of nanoparticles by controlling their shape and size during and after a growth process. Another goal is to motivate future measurements of extinction and absorption spectra of nanoparticles.

\section{Formalism}

In this work, the nanoparticles of interest are typically large enough to accurately apply classical electromagnetic theory to describe their interaction with light [21]. But they are also small enough to observe strong variations in the optical properties depending on the particle size, shape, and local environment. Because of the complexity of the systems being studied, efficient computational methods capable of treating large size materials are essential. In the last few years, several numerical methods have been developed to determine the optical properties of small particles, such as the Discrete Dipole Approximation (DDA), T-matrix and Spectral Representation methods (SR) [22].

In this work we employed the DDA, which is a computational procedure suitable for studying scattering and absorption of electromagnetic radiation by particles with sizes of the order or less of the wavelength of the incident light. DDA has been applied to a broad range of problems, including interstellar and interplanetary dust grains, ice crystals in the atmosphere, human blood cells, surface features of semiconductors, metal nanoparticles and their aggregates, and more. The DDA was first introduced by Purcell and Pennypacker [23], and has been subjected to several improvements, in particular those made by Draine, and collaborators [24]. Below, we briefly describe the main characteristics of DDA and its numerical implementation: the DDSCAT code. For a full description of DDA and DDSCAT, the reader may consult Refs. [21-23].

The main idea behind DDA is to approximate a scatterer, in our case the nanoparticle,

by an large enough array of polarizable point dipoles. Once the location and polarizability of 
each dipole are specified, the calculation of the scattering and absorption efficiencies by the dipole array can be performed, depending only on the accuracy of the mathematical algorithms and the capabilities of the computational hardware. Although the calculation of the radiated fields in DDA is, in principle, also possible, it is actually beyond the computational capabilities of any of the present systems.

\subsection{Discrete Dipole Approximation}

Let us assume an array of $N$ polarizable point dipoles located at $\left\{\mathbf{r}_{i}\right\}, i=1,2, \ldots, N$, each one characterized by a polarizability $\alpha_{i}$. The system is excited by a monochromatic incident plane wave $\mathbf{E}_{\text {inc }}(\mathbf{r}, t)=\mathbf{E}_{0} e^{i \mathbf{k} \cdot \mathbf{r}-i \omega t}$, where $\mathbf{r}$ is the position vector, $t$ is time, $\omega$ is the angular frequency, $k=\omega / c=2 \pi / \lambda$ is the wavevector, $c$ is the speed of light, and $\lambda$ is the wavelength of the incident light. Each dipole of the system is subjected to an electric field that can be split in two contributions: (i) the incident radiation field, plus (ii) the field radiated by all the other induced dipoles. The sum of both fields is the so called local field at each dipole and is given by

$$
\mathbf{E}_{i, \text { loc }}=\mathbf{E}_{i, \text { inc }}+\mathbf{E}_{i, \text { dip }}=\mathbf{E}_{0} e^{i \mathbf{k} \cdot \mathbf{r}_{\mathbf{i}}}-\sum_{i \neq j} \mathbf{A}_{i j} \cdot \mathbf{P}_{j},
$$

where $\mathbf{P}_{i}$ is the dipole moment of the $i$ th-element, and $\mathbf{A}_{i j}$ with $i \neq j$ is an interaction matrix with $3 \times 3$ matrices as elements, such that

$$
\begin{aligned}
\mathbf{A}_{i j} \cdot \mathbf{P}_{j} & =\frac{e^{i k r_{i j}}}{r_{i j}^{3}}\left\{k^{2} \mathbf{r}_{i j} \times\left(\mathbf{r}_{i j} \times \mathbf{P}_{j}\right)\right. \\
& \left.+\frac{\left(1-i k r_{i j}\right)}{r_{i j}^{2}}\left[r_{i j}^{2} \mathbf{P}_{j}-3 \mathbf{r}_{i j}\left(\mathbf{r}_{i j} \cdot \mathbf{P}_{j}\right)\right]\right\}
\end{aligned}
$$

Here $r_{i j}=\left|\mathbf{r}_{i}-\mathbf{r}_{j}\right|$, and $\mathbf{r}_{i j}=\mathbf{r}_{i}-\mathbf{r}_{j}$, and we are using cgs units. Once we solve the $3 N$-coupled complex linear equations given by relation

$$
\mathbf{P}_{i}=\alpha_{i} \cdot \mathbf{E}_{i, \mathrm{loc}}
$$


and determined each dipole moment $\mathbf{P}_{i}$, we can then find the extinction and absorption cross sections for a target, $C_{\text {ext }}$ and $C_{\text {abs }}$, in terms of the dipole moments as

$$
\begin{aligned}
C_{\text {ext }} & =\frac{4 \pi k}{\left|\mathbf{E}_{0}\right|^{2}} \sum_{i=1}^{N} \operatorname{Im}\left(\mathbf{E}_{i, \mathrm{inc}}^{*} \cdot \mathbf{P}_{i}\right) \\
C_{\mathrm{abs}} & =\frac{4 \pi k}{\left|\mathbf{E}_{0}\right|^{2}} \sum_{i=1}^{N}\left\{\operatorname{Im}\left[\mathbf{P}_{i} \cdot\left(\boldsymbol{\alpha}_{i}^{-1}\right)^{*} \mathbf{P}_{i}^{*}\right]-\frac{2}{3} k^{3}\left|\mathbf{P}_{i}\right|^{2}\right\},
\end{aligned}
$$

where $*$ means complex conjugate. The scattering cross section can be obtained using the following relation,

$$
C_{\mathrm{ext}}=C_{\mathrm{sca}}+C_{\mathrm{abs}}
$$

There is some arbitrariness in the construction of the array of dipole points that represent a solid target of a given geometry. For example, the geometry of the grid where the dipoles have to be located is not uniquely determined and a cubic grid is usually chosen. Also, it is not obvious how many dipoles are required to adequately approximate the target, or which is the best choice of the dipole polarizabilities. If one chooses the separation between dipoles $d$ such that $d<<\lambda$, then, one can assign the polarizability for each particle $i$ in vacuum, using the Lattice Dispersion Relation (LDR) polarizability, $\alpha_{i}^{\mathrm{LDR}}$ at a third order in $k$, given by $[24]$

$$
\alpha_{i}^{\mathrm{LDR}}=\frac{\alpha_{i}^{\mathrm{CM}}}{1+\alpha_{i}^{\mathrm{CM}}\left[b_{1}+b_{2} \boldsymbol{\epsilon}_{i}+b_{3} S \boldsymbol{\epsilon}_{i}\right]\left(k^{2} / d\right)},
$$

where $\epsilon_{i}$ is the macroscopic dielectric function of the particle, $\alpha_{i}^{\mathrm{CM}}$ is the polarizability given by the well known Clausius-Mossotti relation,

$$
\alpha_{i}=\left(\frac{d}{3}\right)^{3} \frac{\left(\epsilon_{i}-1\right)}{\left(\epsilon_{i}+2\right)}
$$

and $S, b_{1}, b_{2}$, and $b_{3}$ are coefficients of the expansion.

Now the question is, how many dipoles we need to mimic the continuum macroscopic particle with an array of discrete dipoles? The answer is not straightforward, since we have to consider the convergence of the physical quantities as a function of the dipole number. It has been found that $N \geq 10^{4}$ for an arbitrary geometry is a good starting number, as shown in the Appendix. However, we have a matrix of $(3 N)^{2}$ complex elements which would require a large amount of computational effort. 
In this work we have employed the code adapted by Draine and Flatau to solve the complex linear equations found in DDA. To solve the complex linear equations directly would require tremendous computer capabilities, however, one can use iterative techniques to compute the vector $\mathbf{P} \equiv\left\{\mathbf{P}_{i}\right\}$. In this case, each iteration involves the evaluation of matrixvector products such as $\mathbf{A} \cdot \mathbf{P}^{(\mathrm{n})}$, where $n$ is the number of the iteration. The algorithm, named DDSCAT, locates the dipoles in a periodic cubic lattice, and it is possible to use fast Fourier transform techniques to evaluate matrix-vector products such as $\mathbf{A} \cdot \mathbf{P}$, which allows the whole computation of the final vector $\mathbf{P}$ for a large number of dipoles [25]. For a detailed description of DDA and DDSCAT code, the reader can look at Refs. [22-25].

\section{Results and Discussion}

We define the extinction, absorption and scattering efficiencies or coefficients, $Q_{\text {ext }}, Q_{\text {sca }}$ and Qabs as,

$$
Q_{\mathrm{ext}}=\frac{C_{\mathrm{ext}}}{A}, \quad Q_{\mathrm{abs}}=\frac{C_{\mathrm{abs}}}{A}, \quad Q_{\mathrm{sca}}=\frac{C_{\mathrm{sca}}}{A}
$$

where $A=\pi a_{\text {eff }}^{2}$, and $a_{\text {eff }}$ is defined through the concept of an effective volume equal to $4 \pi a_{\text {eff }}^{3} / 3$. In Figs. 1 to 8 we show $Q_{\text {ext }}, Q_{\text {sca }}$ and $Q_{\text {abs }}$ in dotted-, dashed- and solid-lines, respectively, as a function of the wavelength of the incident light, $\lambda$, for nanometric-size particles. All the calculations were done for nanoparticles with $a_{\text {eff }}=50 \mathrm{~nm}$, and dielectric functions as measured on bulk silver and gold by Johnson and Christy [26]. The nanoparticles were represented or mimic by around 65,000 point-dipoles in order to have a good convergence on their optical properties, as we will discuss below. This number of dipoles is quite large in comparison with the numbers used in previous studies on isolated and supported small metallic nanoparticles $[17,18]$ where, incidentally, only the extinction efficiency was reported. Our use of a large number of dipoles is in agreement with the results found in a a previous work where we found that even for small metallic nanoparticles with radii of about a few nanometers, one needed more than 12,000 dipoles to achieve convergence on their optical properties [20]. In particular, we found that the extinction efficiency converges very rapidly as a function of the number of dipoles. However, this is not the case for the absorption or scattering efficiencies where a very large number of dipoles were necessary to achieve 
such a convergence. As we show in this paper, to elucidate the optical properties of large nanoparticles it is indispensable to do an in-depth study of the scattering and absorption efficiencies, and not only of the extinction one.

In Fig. 1, we show the optical efficiencies for a sphere of radius of $50 \mathrm{~nm}$. In the spectra we can observe that at about $320 \mathrm{~nm}$ all the efficiencies have a local minimum that corresponds to the wavelength at which the dielectric function of silver, both real and imaginary parts, almost vanish. Therefore, this feature of the spectra is inherent to the material properties and, as we observe below, it is independent of the particle geometry. Below $320 \mathrm{~nm}$, the absorption of light is mainly due to the intra-band electronic transitions of silver, therefore, this feature of the spectra should be also quite independent of the shape and size of the particles, as it is actually corroborated in all the graphs shown below corresponding to a silver particle. At about $350 \mathrm{~nm}$, the spectrum of $Q_{\mathrm{abs}}$ shows a peak that is related to the excitation of the surface plasmon of the sphere, therefore this feature is inherent to the geometry of the particle, although the position depends on its material properties. At larger wavelengths the spectrum of $Q_{\text {abs }}$ shows specific features from $380 \mathrm{~nm}$ to about $500 \mathrm{~nm}$ that corresponds to plasmon excitations due to higher multipolar charge distributions [19]. If we look only at the $Q_{\text {ext }}$ it is not possible to observe such features since scattering effects hide them. The $Q_{\text {sca }}$ spectrum shows a broad structure from $320 \mathrm{~nm}$ to $750 \mathrm{~nm}$, with a maximum at about $400 \mathrm{~nm}$, which is three times more intense than the maximum of $Q_{\mathrm{abs}}$. The characteristics of the $Q_{\text {sca }}$ spectrum are mainly due to the size of the particle. In a previous work [20] we found that this maximum is less pronounced as the radius of the sphere increases. Also, the $Q_{\text {sca }}$ spectra of nanospheres decay slowly as the radius increases; this only means that as the sphere becomes larger it scatters light at longer wavelengths, as expected.

In Fig. 2, we show the optical efficiencies for a nanocube with sides of $83 \mathrm{~nm}$. For wavelengths between $320 \mathrm{~nm}$ and $450 \mathrm{~nm}$ the main contributions to the $Q_{\text {ext }}$ spectrum comes from light absorption and scattering, while for $400 \mathrm{~nm}<\lambda<700 \mathrm{~nm}$, it comes mainly from light scattering effects. The $Q_{\text {abs }}$ spectrum shows a rich structure for $\lambda$ between $320 \mathrm{~nm}$ and $420 \mathrm{~nm}$, where several peaks are observed. These peaks are associated to the resonances inherent to the cubic geometry [27]. The peak at $\lambda=410 \mathrm{~nm}$ corresponds to 
the dipolar resonance, while the peaks at smaller wavelengths are due to high-multipolar excitations. From $420 \mathrm{~nm}$ to about $700 \mathrm{~nm}, Q_{\text {abs }}$ shows a tail that corresponds also to high-multipolar excitations, however, these excitations are due to size effects. Therefore, this tail is observed in all figures since all the nanoparticles have the same volume. We also performed calculations for silver cubes with sides of $9 \mathrm{~nm}$, where we found that for $\lambda<410 \mathrm{~nm}$ the $Q_{\mathrm{abs}}$ spectrum has more or less the same structure and intensity than the one shown here, but for larger wavelengths it does not have a tail. Above the resonances, at about $\lambda=450 \mathrm{~nm}$, the $Q_{\text {sca }}$ spectrum shows a maximum. We can observe that this maximum is at a larger wavelength than the one corresponding to a sphere. This shift of the scattering peak to larger wavelengths is due to the increase in the size of the nanoparticle. The latter observation was also described in a previous work [20], where the $Q_{\text {ext }}$ and $Q_{\text {abs }}$ spectra for nanocubes of $a_{\text {eff }}=50 \mathrm{~nm}$ and $a_{\text {eff }}=150 \mathrm{~nm}$, were compared. In Fig. 8, we will display the optical efficiencies of a gold cube of the same size to discuss the effects due to material properties.

In Fig. 3 we show the optical efficiencies of a silver prolate spheroidal nanoparticle where the incident electromagnetic field is taken parallel to its minor axis. The spheroid has a major to minor axis ratio of 3 to 1 . From $320 \mathrm{~nm}$ to about $450 \mathrm{~nm}$ one can see that the contributions to the $Q_{\text {ext }}$ spectrum from absorption and scattering processes are more or less of the same order of magnitude. Notice that the peak at shorter wavelengths $(\sim 350 \mathrm{~nm})$ is almost twice as big as the peak at longer wavelengths $(\sim 430 \mathrm{~nm})$. The $Q_{\text {abs }}$ spectrum is characterized by two peaks, one at $\sim 350 \mathrm{~nm}$, and the other at $\sim 430 \mathrm{~nm}$, and a tail from $500 \mathrm{~nm}$ to $750 \mathrm{~nm}$. The first peak corresponds to the main resonance of an ellipsoid due to a dipolar charge distribution and its position depends on the specific geometry of the particle. On the other hand, the peak and the tail at longer wavelengths correspond to excitations of higher multipolar charge distributions. We recall that although the DDA cannot assign a specific multipolar order to those excitations [19], one can assert that in this particular geometry, the high multipolar excitations are due to size effects because they are not observed in small spheroidal nanoparticles. Furthermore, it is necessary to do an in-depth study of both absorption and scattering effects to clearly elucidate the problem. For example, it is known that scattering effects of smaller particles have a maximum at smaller 
wavelengths, therefore it is possible to hide the multipolar effects if they appear at the same wavelength as scattering effects do. In such case, a detail study of the absorption and extinction efficiencies is necessary. We have also calculated the optical efficiencies for smaller ellipsoidal nanoparticles with a major semiaxis of $12 \mathrm{~nm}$. In this case, the main contribution to $Q_{\text {ext }}$ comes from light absorption processes due to the excitation of one surface plasmon (dipolar excitation) whose location depends on the particular geometry of each nanoparticle and on its material properties. From $475 \mathrm{~nm}$ to $750 \mathrm{~nm}$ the main contribution to $Q_{\text {ext }}$ come from light-scattering effects, although, we also observed a tail in $Q_{\text {abs. }}$. Although this tail shows also a few peaks, they are washed out as the number of dipoles in the calculation is increased dramatically [20], so they should come from lack of convergence in the calculations. It is interesting to note that this lack of convergence is particularly observed in metal particles and it could be due to the fact that at long wavelengths the dielectric function of silver, and in general of metals, is negative and large [28].

In Fig. 4 we show the optical efficiencies for the same silver spheroidal nanoparticle but now with the incident electromagnetic field perpendicular to its minor axis. Like for the previous orientation, the spectrum of $Q_{\text {abs }}$ shows also two peaks and a tail, however the peak at $350 \mathrm{~nm}$ is less intense than the peak at about $430 \mathrm{~nm}$. In this case, the dipolar excitation or main surface plasmon is observed at $\lambda=430 \mathrm{~nm}$, while high-multipolar excitations are observed at $\lambda=350 \mathrm{~nm}$. These changes in the excitation energy of the multipolar modes depending on the orientation of the polarization of the incident light have been already calculated for oblate and prolate spheroids using the exact solution in the quasi-static limit, where multipolar excitations are induced by the presence of a substrate [14]. Here the $Q_{\text {ext }}$ spectrum has again contributions from both absorption and scattering processes. From $475 \mathrm{~nm}$ to $750 \mathrm{~nm}$ the main contribution to $Q_{\text {ext }}$ comes from light scattering effects, as it does in Fig. 3. However, for this orientation of the ellipsoid $Q_{\text {ext }}$ is about twice as intense as the one corresponding to an incident electromagnetic field parallel to the major axis. Besides that, it is possible to assign a particular geometry to each spectra, so we can also obtain information about its orientation. However, since the intensity and sharpness of the spectra is dominated by the material properties, it is possible that this kind of information could be hidden if the dielectric response of the particle is different [20]. 
In Fig. 5 we show the optical efficiencies for a silver cylinder when the incident electromagnetic field is parallel to its symmetry axis. The cylinder has a radius of $43.7 \mathrm{~nm}$ and its axis is two times larger than its radius. From $320 \mathrm{~nm}$ to $470 \mathrm{~nm}$ the absorption effects are as important as the light scattering effects. The $Q_{\text {abs }}$ spectrum shows three main peaks which are due to the geometrical properties of the nanoparticle, as we have discussed before. The $Q_{\text {abs }}$ spectrum also shows a tail at larger wavelengths due to high-multipolar excitations coming from size effects. In $Q_{\text {abs }}$ the peak at $\lambda \sim 430 \mathrm{~nm}$ corresponds to a dipolar excitations, while the other two peaks at lower wavelengths are due to multipolar excitations inherent to the geometry of the particle. These multipolar excitations are enhanced due to size effects, since for smaller nanocylinders we observe that the dipolar excitation is fourteen times more intense than other multipolar excitations. At longer wavelengths the spectrum is dominated by scattering effects, as shown in the figure. For this geometry, we observe that the scattering processes are very efficient as compared with the corresponding ones in other particles, and the main peak in the extinction spectrum is located at longer wavelengths. This geometry is similar to the spheroidal nanoparticles, therefore, we observed that both spectra are also similar, except that the absorption of the cylinder shows an additional resonance.

In Fig. 6 we show the optical efficiencies of a silver tetrahedral nanoparticle when the incident electric field is parallel to its basis, and it is pointing towards one of its vertex. The tetrahedron has sides of $164 \mathrm{~nm}$. The spectra for $\lambda>320 \mathrm{~nm}$ are different from those discussed above, since a more complex resonance structure is found. In this case, light absorption and scattering occur over a wide range of wavelengths. For $\lambda<500 \mathrm{~nm}$ the absorption effects are a little more intense than scattering effects, and for $\lambda>500 \mathrm{~nm}$ the contrary occurs. For this particular geometry, we observe that the spectra have a very rich structure coming from the excitation of surface plasmons as well as from light scattering. The $Q_{\text {abs }}$ spectrum has a dipolar resonance at about $500 \mathrm{~nm}$ which is enhanced by multipolar excitations from both geometry and size effects. At smaller wavelengths, the $Q_{\text {abs }}$ spectrum is dominated by high-multipolar excitations due to the geometry of the particle. These high-multipolar excitations have been experimentally observed and theoretically identified for $\mathrm{Ag}$ prisms $[29,30]$. The $Q_{\text {sca }}$ spectrum shows a maximum at about $\lambda=590 \mathrm{~nm}$. Notice that from $600 \mathrm{~nm}$ to $750 \mathrm{~nm}$ the $Q_{\text {abs }}$ spectrum shows several peaks coming from lack of 
convergence in the calculations, thus they have no physical meaning as it was pointed out before.

In Fig. 7 we show the optical efficiencies for a silver nanoparticle that is made of small spheres which are so arranged as to resemble a kind of pyramid with a square base. The sides of the base of the pyramid is $146 \mathrm{~nm}$ while its high is $73 \mathrm{~nm}$. We have taken the incident electric field parallel to its base. Like for the tetrahedron, the spectra are completely different from those corresponding to the other silver nanoparticles discussed above. When $\lambda>320 \mathrm{~nm}$ light absorption and scattering occur over a wide range of wavelengths, being the absorption effects the ones that dominate the structure of the spectrum. The spectra of the pyramid also have a very rich structure coming from the excitation of surface plasmons as well as from the light scattering corresponding to this particular geometry. However, the structure of the $Q_{\text {ext }}$ is rather smooth as compared to the one of the tetrahedral nanoparticle. This could be associated to the fact that the pyramid is constructed by using small spheres, and each sphere has a single surface plasmon and a well defined scattering peak. However, in this case it was not possible to distinguish between dipolar and high-multipolar excitations. Again, we observe four sharp peaks at large wavelengths which come from lack of convergence in the calculations at those specific wavelengths, nevertheless, the spectra should be valid at all the other wavelengths. The enhancement of these peaks could come from the fact that the small spheres are touching, however a more careful study has to be done.

In Fig. 8 we show the optical efficiencies for a nanocube with the same volume as the one in Fig. 2, but made out of gold. For the gold cube the $Q_{\text {ext }}$ spectrum shows a broad structure from $250 \mathrm{~nm}$ to about $500 \mathrm{~nm}$, due mainly to absorption effects although light scattering is also observed. Contrary to the rich structure found for the silver cube, in this case we observe very smooth curves. This behavior comes from the fact that the relaxation time of electrons in gold is about ten times smaller than the relaxation time for silver, this fact gives rise to a completely different dielectric response. The several peaks found for the silver nanoparticles are now replaced by a wide structure that is due to surface plasmons as well as intra-band transitions. Then, we can conclude that both effects are of the same order of magnitude since the fine structure inherent to a cube has been washed out. The latter gives rise to a wide peak in $Q_{\text {abs }}$ at about $550 \mathrm{~nm}$ and a peak in $Q_{\text {sca }}$ at about $580 \mathrm{~nm}$. 
Notice that at $\lambda=580 \mathrm{~nm}$ the $Q_{\text {sca }}$ spectra of the silver (Fig. 2) and gold (Fig. 8) nanocubes are of the same magnitude. However for the silver cube the $Q_{\text {sca }}$ has its maximum at lower wavelengths and it is almost twice as large as the one corresponding to the gold cube, where its maximum seems to be inhibited and this might be due to intra-band transitions.

Recently silver and gold suspended nanoparticles have been produced by wet chemistry techniques [29] and by laser ablation in aqueous solution [31, 32]. In the first case, the nanoparticles have been fully characterized by measurements of the extinction efficiency [29]. In the second case, the authors reported the production of gold nanoparticles with a diameter between $1 \mathrm{~nm}$ to $50 \mathrm{~nm}$, as well as silver nanoparticles with diameters between $1 \mathrm{~nm}$ to $40 \mathrm{~nm}$. The reported absorption spectrum of silver nanoparticles show a minimum at about $320 \mathrm{~nm}$, and a wide peak with a maximum around $400 \mathrm{~nm}$, and at about $600 \mathrm{~nm}$ the spectrum starts to decay very fast. On the other hand, for gold nanoparticles the absorption spectrum shows a wider structure from $300 \mathrm{~nm}$ to $800 \mathrm{~nm}$, showing a shoulder at about $520 \mathrm{~nm}$. The authors argued that such nanoparticles have spherical geometry, however, we believe that it is difficult assert because the absorption peak is so wide in both cases that any particular optical signature due to geometry might be hidden by the distribution of sizes and shapes of the nanoparticles, and not only due to the effects in the change of the electron mean free path or in the absorption energies of intra-band transitions, as it was argued [31]. Unfortunately a direct comparison between our calculation and their measurements is not possible since their samples might have a large distribution of sizes, as well as a large variety of shapes.

\section{Conclusions}

We use the discrete dipole approximation to study the main optical features of the extinction, absorption and scattering efficiencies of nanoparticles of different sizes and shapes, made out of silver and gold. Here we have considered different geometries: spheres, ellipsoids, cubes, tetrahedra, cylinders and pyramids. In most cases, we have clearly identified the main optical signature associated to each geometry. We find, as it might have been expected, that the spectra are more complex as the particle has less symmetry and /or has more vertices. We

have identified in the spectra the main surface plasmon resonance associated to a dipolar 
excitation, as well as other resonances due to high-multipolar excitations. The physical origin of these high-multipolar excitations might have two different sources, one due to the shape and the other due to the size of the particle. In the case of silver particles, specific features in the optical spectra can be associated to either geometry, size or material properties, making optical spectroscopies a very helpful tool for the characterization of nanoparticles during and after growth. We also show that it is not enough to analyze the extinction efficiency to elucidate the size and shape of a nanoparticle, but that is absolutely necessary to do also an in-depth study of the absorption and scattering efficiencies.

A direct comparison of our results with most of the available experimental measurements of the optical properties of suspended nanoparticles would require an averaging procedure over a wide distribution of sizes and shapes. But this averaging procedure might smooth out the main relevant features of the spectra associated to the size and shape of the nanoparticles. On the other hand, it would be very desirable to obtain optical spectra over samples with narrower distributions of sizes and shapes.

We appreciate valuable discussions with Prof. Yves Borensztein and Guillermo Ortiz. This work has been partly supported by DGAPA-UNAM grant No. IN104201 and by CONACyT grants 36651-E and G32723-E.

\section{A Surface effects}

One criteria to choose the number of dipoles or the separation distance between them is to avoid spurious surface effects. We call spurious surface effects to those effects coming from the fact that for a given cubic lattice bounded by a specific particle shape, it is possible to have a number of dipoles at the surface which can be larger or comparable to the number of dipole within the particle.

Let us assume that we have a sphere of volume $V$ which can be discretized in $\mathrm{N}$ spherical entities with radius $d / 2$, where $d$ is the separation between dipoles. We have that $V \simeq$ $N v=4 \pi a_{\text {eff }}^{3} / 3$, where $v$ is the volume ocuppied by each dipole $v=4 \pi(d / 2)^{3} / 3$, and $a_{\text {eff }}$ is

the effective radius of the particle. Now, we want to know how many dipoles belong to the 
surface. Suppose we have $N_{s}$ dipoles at the surface which occupies a volume $N_{s} v$,

$$
N_{s} v=\frac{4 \pi}{3} a_{\mathrm{eff}}^{3}-\frac{4 \pi}{3}\left(a_{\mathrm{eff}}-d / 2\right)^{3}
$$

where we find that

$$
N_{s}=N\left[1-\frac{1}{N}\left(N^{1 / 3}-1\right)^{3}\right]
$$

Using this formula, we have that for a total of $N=100$ dipoles, about 52 of them belong to the surface, for $N=1000$, about 271 dipoles belong to the surface, and so on, as shown in Table 1.

\section{References}

[1] See for example, Mater. Res. Bull. 1998, 23, 31; and references therein.

[2] M. José Yacamán, J.A. Ascencio, H.B. Liu, J. Gardea-Torresdey, J. Vac. Sci. Technol. B 2001, 19, 1091.

[3] S. Ijima and H. Ichihashi, Phys. Rev. Lett. 1986, 56, 616.

[4] J. O. Bovin, R. L. Wallember, and D. Smith, Nature 1985, 317, 47.

[5] B. Damilano, N. Grandjean, S. Vézian and J. Massies, J. of Crystal Growth, 2001, 227, 466.

[6] M.J. Zheng, X. Y. Zhang, L. Yang, C. H. Liang and L. D. Zhang, Semicond. Sci. Technol 2001, 16, 507 .

[7] T. W. Kim, D. U. Lee, D. C. Choo, H. J. Kim, H. S. Lee, J. Y. Lee, M. D. Kim, S. H. Park and H. L. Park, Solid State Comm. 2001, 118, 465.

[8] G. E. Cirlin, et al., Material Science and Engineering B, 2001, 80, 108.

[9] N. Liu, J. Tersoff, O. Baklenov, A.L. Holmes, Jr., and C.K. Shih, Phys. Rev. Lett. 2000, 84,334 .

[10] J. Zou, X.Z. Liao, D.J.H. Cockayne, and R. Leon, Phys. Rev. B 1999, 59, 12279. 
[11] W. Yang, H. Lee, T.J. Johnson, P.C. Sercel, and A.G. Norman, Phys. Rev. B 2000, 61,2784 .

[12] C. E. Román-Velázquez, C. Noguez, and R.G. Barrera, MRS Symposium Proceedings 2000, 581, 485; Editors S. Komarneni, J.C. Parker, H. Hahn.

[13] C. Beitia, Y. Borensztein, R. G. Barrera, C. E. Román, C. Noguez, Physica B 2000, $279,25$.

[14] C. E. Román, C. Noguez, and R.G. Barrera, Phys. Rev. B 2000, 61, 10427.

[15] See for example: A. R. Turner, M. E. Pemble, J. M. Fernández, B. A. Joyce, J. Zhang, and A. G. Taylor, Phys. Rev. Lett. 1995, 74, 3213; C. Noguez, C. Beitia, W. Preyss, A.I. Shkrebtii, M. Roy, Y. Borensztein and R. Del Sole, Phys. Rev. Lett. 1996, 76, 4923; B. G. Frederick, J.R. Power, R.J. Cole, C.C. Perry, Q. Chen, S. Haq, Th. Bertrams, N. V. Richardson, and P. Weightman, Phys. Rev. Lett. 1998, 80, 4490; J. R. Power, P. Weightman, S. Bose, A.I. Shkrebtii, and R. Del Sole, Phys. Rev. Lett.1998, $80,3133$.

[16] N. Félidj, J. Aubard, and G. Lévi, J. Chem. Phys. 1999, 111, 1195.

[17] W.-H. Yang, G. C. Schatz, and R. P. van Duyne, J. Chem. Phys. 1995, $103,869$.

[18] T. R. Jensen, M. L. Duval, K. L. Kelly, A. Lazarides, G. C. Schatz, R. P. Van Duyne, J. Phys. Chem. B 1999, $103,9846$.

[19] Unfortunately, using DDA it is not a direct method to assign a specific multipolar order to a given resonance structure. This is because the near field around the particle is not well described in this approximation. This has been pointed out before in Ref. 21.

[20] C. Noguez, I. Sosa, and R. G. Barrera, MRS Proceedings 2002, 704, 275; Editors: R.K. Singh, R. Partch, M. Muhammed, M. Senna, H. Hofmann.

[21] K. Lance Kelly, A.A. Lazarides, and G.C. Schatz, Computing in Science and Engineering, 2001, 3, 67 . 
[22] Light Scattering by Nonspherical Particles, Edited by M. I. Mishchenko, J. W. Hovenier, and L. D. Travis, Academic Press, San Diego, 2000.

[23] E.M. Purcell and C.R. Pennypacker, Astrophys. J. 1973, 186, 705.

[24] B. T. Draine, Astrophys. J. 1998, 333, 848; B. T. Draine and J.J. Goodman, Astrophys. J. 1993, 405, 685; B. T. Draine and P.J. Flatau, J. Opt. Am. A 1994, 11, 1491.

[25] Program DDSCAT, by B. T. Draine and P.J. Flatau, University of California at San Diego.

[26] P. B. Johnson and R. W. Christy, Phys. Rev. B 1972, 6, 4370.

[27] R. Fuchs, Phys. Rev. B 1975, 11, 1732.

[28] We performed calculations of absortion and scattering effciencies with metal nanoparticles described by the Drude model using different plasma frequencies $\left(\omega_{p}\right)$ and relaxation times $(\tau)$. In all cases, independently of $\omega_{p}$ and $\tau$, we observed that for large dielectric functions convergency problems are always present. On the other hand, we have also performed calculations with semiconductor nanoparticles and this problem of convergency is not observed. We have also found that if the number of dipoles are increased dramatically from 65,000 to half a million, then the optical properties converges.

[29] R. Jin, Y. Cao, C.A. Mirkin, K.L. Kelly, G.C. Schatz, J.-G. Zheng, Science 2001, 294, 1901.

[30] K.L. Kelly, E. Coronado, L.L. Zhao, G.C. Schatz, J. Phys. Chem. B 2003, 10 \%, 668.

[31] F. Mafuné, J. Kohno, Y. Takeda, T.Kondow and H. Sawabe, J. Phys. Chem. B 2000, 104, 8333; J. Phys. Chem. B 2000, 104, 9111; J. Phys. Chem. B 2001, 105, 5114.

[32] F. Mafuné, J. Kohno, Y. Takeda, and T.Kondow, J. Phys. Chem. B 2001, 105, 9050; J. Phys. Chem. B 2002, 106, 7575; J. Phys. Chem. B 2002, 106, 8555. 
Table 1: Number of surface dipoles according to Eq. A2

\begin{tabular}{c|c|c}
\hline \hline total dipoles $N$ & surface dipoles $N_{s}$ & $N / N_{s}(\%)$ \\
\hline $10^{2}$ & 51.7 & 51.7 \\
$10^{3}$ & 271.0 & 27.1 \\
$10^{4}$ & 1328.8 & 13.2 \\
$10^{5}$ & 6325.0 & 6.32 \\
$10^{6}$ & 29701.0 & 2.97 \\
\hline \hline
\end{tabular}




\section{FIGURE CAPTIONS}

FIGURE 1: Optical coefficients for a silver nanosphere

FIGURE 2: Optical coefficients for a silver nanocube

FIGURE 3: Optical coefficients for a silver nanospheroid for an electric field polarized along the minor axis

FIGURE 4: Optical coefficients for a silver nanospheroid for an electric field polarized along the major axis

FIGURE 5: Optical coefficients for a silver nanocylinder for an incident electromagnetic field along the symmetry axis

FIGURE 6: Optical coefficients for a silver tetrahedra for an incident electromagnetic field perpendicular to the basis

FIGURE 7: Optical coefficients for a silver piramide for an incident electromagnetic field perpendicular to the basis

FIGURE 8: Optical coefficients for a gold nanocube 
This figure "fig1.jpg" is available in "jpg" format from: http://arxiv.org/ps/cond-mat/0304216v1 
This figure "fig2.jpg" is available in "jpg" format from: http://arxiv.org/ps/cond-mat/0304216v1 
This figure "fig3.jpg" is available in "jpg" format from: http://arxiv.org/ps/cond-mat/0304216v1 
This figure "fig4.jpg" is available in "jpg" format from: http://arxiv.org/ps/cond-mat/0304216v1 
This figure "fig5.jpg" is available in "jpg" format from: http://arxiv.org/ps/cond-mat/0304216v1 
This figure "fig6.jpg" is available in "jpg" format from: http://arxiv.org/ps/cond-mat/0304216v1 
This figure "fig7.jpg" is available in "jpg" format from: http://arxiv.org/ps/cond-mat/0304216v1 
This figure "fig8.jpg" is available in "jpg" format from: http://arxiv.org/ps/cond-mat/0304216v1 